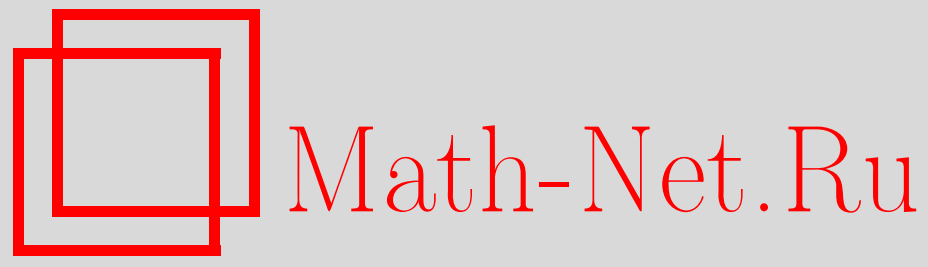

О. Г. Парфенов, Асимптотика поперечников по Гельфанду и Бернштейну некоторых классов аналитических функций, Функи. анализ и его прил., 1996, том 30, выпуск 1, 79-80

DOI: https://doi.org/10.4213/faa512

Использование Общероссийского математического портала MathNet.Ru подразумевает, что вы прочитали и согласны с пользовательским соглашением

http://www. mathnet.ru/rus/agreement

Параметры загрузки:

IP : 44.207 .124 .84

26 апреля 2023 г., 15:47:09

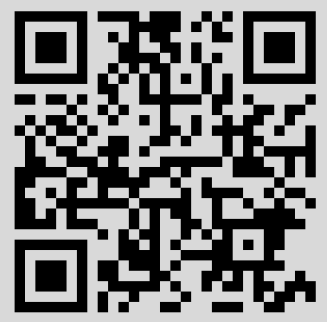


УдК $517.47+517.51$

\title{
Асимптотика поперечников по Гельфанду и Бернштейну некоторых классов аналитических функций
}

\author{
(C) 1996. О. Г. ПАРФЕНОВ
}

1. Пусть $H^{p}, 1 \leqslant p \leqslant \infty$, - класс Харди в единичном круге $\mathbb{D}, T_{r}=$ $\{z:|z|=r\}, 0<r \leqslant 1$, и $\mathbb{T}=T_{1}$. В работах автора $[1,2]$ исследовались поперечники по Гельфанду единичного шара $B H^{p}$ в метрике пространства $L_{q}\left(T_{r}, \mu\right)$ при $p \geqslant q$, где $\mu$ - произвольная мера на $T_{r}$.

В работе Фишера и Стесина [3] показано, что при $p \geqslant q$ поперечники по Гельфанду единичного шара $B H^{p}$ в метрике пространства $L_{q}(K, \mu), K-$ некоторый компакт в $\mathbb{D}$, совпадают с колмогоровскими и линейными. При $p<q$ пока известно немного об интересующих нас поперечниках (см., например, [4]). В работе автора [5] получены теоремы типа Исмагилова для поперечников по Гельфанду и Бернштейну. Сопоставляя результаты работ [1] и [5], можно получить нетривиальные асимптотические оценки снизу для поперечников по Гельфанду и сверху для поперечников по Бернштейну. Получить обратные оценки на основе результатов $[1,5]$ затруднительно. Данная работа и посвящена получению этих оценок. Автор пользуется случаем, чтобы выразить благодарность Российской академии наук за поддержку работы (грант РФФИ 95-01-00348).

2. Пусть $\nu$ - некоторая мера на единичной окружности $\mathbb{T}$ и $m-$ нормированная мера Лебега на $\mathbb{T}$. Положим

$$
G(\nu)=\exp \left(\int_{\mathbb{T}} \ln (d \nu / d m) d m\right) .
$$

Обозначим через $H^{2}(\nu)$ весовой класс Харди, т. е. замыкание множества многочленов в пространстве $L_{2}(\mathbb{T}, \nu)$.

Имеет место следующая теорема:

Теорема 1. Пусть $G(\nu) \neq 0$. Тогда справедливо соотношение ${ }^{1}$

$$
d^{n}(\nu) \equiv d^{n}\left(B H^{2}(\nu), L_{\infty}\left(T_{r}\right)\right) \sim G^{-1 / 2}(\nu)\left(1-r^{2}\right)^{-1 / 2} r^{n}, \quad n \rightarrow \infty .
$$

Поясним основные идеи доказательства теоремы 1. Для оценки сверху введем оператор вложения $J_{0}: H^{2}(\nu) \rightarrow L_{2}\left(T_{r}\right)$. В силу [1] имеем асимптотическую формулу для сингулярных чисел:

$$
s_{n}\left(J_{0}\right) \sim G^{-1 / 2}(\nu) r^{n}, \quad n \rightarrow \infty .
$$

В силу результатов работы [5] имеем

$$
d^{n}(\nu) \geqslant\left(\sum_{k=n}^{\infty} s_{k}^{2}\left(J_{0}\right)\right)^{1 / 2} .
$$

${ }^{1} a_{n} \sim b_{n}, n \rightarrow \infty \Longleftrightarrow \lim a_{n} / b_{n}=1, n \rightarrow \infty$. 
Из (2) и (3) следует требуемая асимптотическая оценка снизу в (1). Для доказательства оценки сверху рассмотрим набор подпространств $\left\{B_{n} \cdot H^{2}(\nu)\right\}, B_{n}$ - произведение Бляшке степени $n$. Имеем

$$
d^{n}(\nu) \leqslant \inf _{B_{n}} \sup _{f \in B H^{2}(\nu)}\left\|B_{n} f\right\|_{L_{\infty}\left(T_{r}\right)} .
$$

Используя формулу для нормы оператора, действующего из гильбертова пространства в $L_{\infty}\left(T_{r}\right)$ (см. [5]) и формулу для предельного ядра (см. [6, с. 70]), получим

$$
\left(d^{n}(\nu)\right)^{2} \leqslant\left(1-r^{2}\right)^{-1} \inf _{B_{n}} \sup _{|z|=r}\left|B_{n}(z)\right|^{2}\left|g_{\nu}(z)\right|^{-2} ;
$$

здесь $g_{\nu}$ - функция Сегё меры $\nu$ (см. [6, с. 37-40]). Из леммы об аппроксимации непрерывных положительных функций модулями произведений Бляшке [1] получим

$$
\inf _{B_{n}} \sup _{|z|=r}\left|B_{n}(z)\right|^{2}\left|g_{\nu}(z)\right|^{-2} \sim G^{-1}(\nu) r^{2 n}, \quad n \rightarrow \infty .
$$

Оценки (4)-(6) и дают в совокупности асимптотическую оценку сверху в теореме 1 . В качестве следствия отметим такой результат:

Теорема 2. Пусть $\mathbb{K}: L_{1}(T) \rightarrow L_{2}\left(T_{r}, \mu\right), G(\mu) \neq 0$, - интегральный оператор с ядром Коши:

$$
(\mathbb{K} f)(z)=\frac{1}{2 \pi i} \int_{\mathbb{T}} \frac{f(w)}{w-z} d w .
$$

Тогда для поперечников по Колмогорову оператора $\mathbb{K}$ имеем

$$
d_{n}(\mathbb{K}) \sim G^{1 / 2}(\mu)\left(1-r^{2}\right)^{-1 / 2} r^{n}, \quad n \rightarrow \infty .
$$

Подчеркнем, что мера $\mu$ произвольна (при условии, что $G(\mu) \neq 0$ ).

3. Для поперечников по Бернштейну имеем следующий результат:

Теорема 3. Пусть $\mu-$ произвольная мера на $T_{r}, G(\mu) \neq 0$. Тогда

$$
b_{n}(\mu) \equiv b_{n}\left(B H^{\infty}, L_{2}\left(T_{r}, \mu\right)\right) \sim G^{1 / 2}(\mu)\left(1-r^{2}\right)^{1 / 2} r^{n}, \quad n \rightarrow \infty .
$$

Доказательство теоремы 3 очень похоже на доказательство теоремы 1 , и мы его опускаем.

\section{ЛитеРАтУРА}

1. Парфенов О. Г. Матем. сб., 117 (159), № 2, 279-285 (1982). 2. Парфенов О. Г. Матем. заметки, 37, № 2, 171-175 (1985). 3. Fisher S., Stessin M. The $n$-widths of unit ball of $H^{q}$. Preprint, Northwestern University, Evanston (1990). 4. Парфенов О. Г. Матем. заметки, 26, вып. 3, 85-87 (1992). 5. Парфенов О. Г. Зап. научн. семинаров ЛОМИ, 217, 112-129 (1994). 6. Гренандер У., Сегё Г. Теплицевы формы и их приложения. ИЛ, М. (1961). 